\title{
AD ASTRA: the database of Allelic Dosage-corrected Allele- Specific TRAnscription factor binding suggests causal regulatory sequence variants of pathologies
}

\author{
Sergey Abramov \\ Vavilov Institute of General Genetics \\ Russian Academy of Sciences \\ Moscow, Russia \\ Abramov.SA@phystech.edu \\ Eugene Baulin \\ Institute of Mathematical Problems of \\ Biology RAS - the Branch of Keldysh \\ Institute of Applied Mathematics of \\ Russian Academy of Sciences \\ Pushchino, Russia \\ baulin@lpm.org.ru \\ Vsevolod J Makeev \\ Vavilov Institute of General Genetics \\ Russian Academy of Sciences \\ Moscow, Russia \\ vsevolod.makeev@vigg.ru
}

\author{
Alexandr Boytsov \\ Vavilov Institute of General Genetics \\ Russian Academy of Sciences \\ Moscow, Russia \\ boytsovs.av@phystech.edu
}

\author{
Ivan Yevshin \\ BIOSOFT.RU LLC \\ Novosibirsk, Russia \\ ivan@developmentontheedge.com \\ Ivan V Kulakovskiy \\ Engelhardt Institute of Molecular \\ Biology \\ Moscow, Russia \\ ivan.kulakovskiy@gmail.com
}

\author{
Bykova Dariia \\ Faculty of Bioengineering and \\ Bioinformatics \\ Lomonosov Moscow State University \\ Moscow, Russia \\ db.bykova@gmail.com
}

\author{
Fedor Kolpakov \\ Institute of Computational \\ Technologies SB RAS \\ Novosibirsk, Russia \\ fkolpakov@gmail.com
}

\begin{abstract}
We present a comprehensive meta-analysis of more than 7 thousand ChIP-Seq experiments that allowed the identification of more than $\mathbf{1 0 0}$ thousand allele-specific binding sites across human transcription factors and cell types. Particularly, the allele-specific binding was found at many regulatory variants having known associations with various pathologies.
\end{abstract}

Keywords - Transcription Factors, ChIP-Seq, Allele-Specific Binding, Allelic Dosage, Phenotypic Traits, Single-Nucleotide Variants, SNV, Single-Nucleotide Polymorphisms, SNP

\section{Introduction}

Sequence variants in genomic regulatory regions act as a source of many hereditary phenotypic traits including predisposition to various pathologies. Variants in promoters and enhancers may affect gene expression regulation at the transcription level by altering transcription factor (TF) binding. Chromatin immunoprecipitation followed by deep sequencing (ChIP-Seq) with enough sequencing depth allows detecting events of allele-specific binding (ASB) i.e. differential TF affinity to alternative alleles of a particular single-nucleotide variant, existing in a heterozygous state in a given cell type. However, most of the published ChIP-Seq data is obtained in cell types with complex aneuploidy and multiple copy-number variants (CNVs), complicating statistical assessment of ASB events due to biased read counts from alternative alleles arising from non-equal background allelic dosage (BAD, the combination of chromosome ploidy and CNV effects).

\section{Results}

We present a new computational approach to ASB calling by accounting for BAD through de novo identification of chromosome duplications and CNVs directly from the read coverage at alternative alleles in aggregated data for each particular cell type. We successfully validated the algorithm using data on copy numbers in immortalized cell lines from the COSMIC database. We also introduced a new statistical approach to estimating ASB significance accounting for the read mapping bias by separately assessing read frequencies at the reference and alternative alleles with the negative binomial test.

We have applied the pipeline for more than 7 thousand ChIP-Seq read alignments available in the GTRD database, identified nearly 35 million candidate single-nucleotide variants with coverage of no less than 10, systematically assessed BAD in more than five hundred of cell types, and found more than $100 \mathrm{~K}$ ASB events with coverage no less than 30 and $5 \%$ FDR.

We have annotated the ASBs by using existing public genotype-phenotype association databases, such as EBI GWAS Catalog, and ClinVar, and identified enriched association of ASB SNPs with different pathologies. Particularly, higher enrichment was detected for ASBs of multiple TFs, suggesting that rewiring of a regulatory network has greater potential to be deleterious compared to disruption or creation of individual regulatory sites.

\section{ACKNOWLEDGMENT}

This study was supported by the Russian Foundation for Basic Research grant 18-34-20024. 